\title{
Relación entre el Parque de Tractores Agrícolas y el Patrón de Difusión y Adopción mediante un Modelo Logístico
}

\author{
Ricardo M. Muñoz*, José L. Llanos y Luis D. Sáez \\ Departamento de Gestión Agraria, Universidad de Santiago de Chile (USACH). \\ Avda. Ecuador 3769, Estación Central, Santiago-Chile (e-mail: ricardo.munoz@usach.cl, \\ jose.llanos@usach.cl, luis.saez@usach.cl) \\ * Autor a quien se debe dirigir la correspondencia
}

Recibido Ene. 26, 2011; Aceptado Mar. 21, 2011; Versión Final recibida May. 24, 2011

\section{Resumen}

Este estudio determina el parque de tractores y la evolución del crecimiento de la adopción agregada del tractor agrícola en Chile. Se aplicó un modelo logístico para determinar el crecimiento de la adopción y la rapidez de adopción del tractor agrícola. Los parámetros del modelo se determinaron mediante un procedimiento no lineal. Se usó el balance del parque de tractores y la curva de tractorización y de adopción para estimar la variación de las unidades entre t y t-1 y se relacionó con la incorporación de tractores y con aquellos dados de baja. El modelo logístico mostró un nivel de ajuste significativo y permitió estimar la tasa de adopción y el nivel de saturación de la adopción. Se concluye que la metodología se puede aplicar para el estudio de la adopción de tecnologías en otras regiones y otros países.

\section{Relationship between the Agricultural Tractor Stock and the Adoption and Diffusion Patterns using a Logistic Model}

\begin{abstract}
This paper determines the tractor stock and aggregate adoption growth of agricultural tractors in Chile. A logistic model was applied to determine the adoption growth and the adoption speed of agricultural tractors. Model parameters were determined by a non-linear algorithm. The tractor stock balance and the tractorization curve were used to estimate variation rate between $t$ and $t-1$. This variation was associated with the introduction of tractors and those units removed from the stock. The logistic model showed a significant adjustment level and allowed estimating the rate of adoption and the adoption saturation level. It is concluded that the methodology can be applied to study technological adoption in other regions and other countries.
\end{abstract}

Keywords: tractorization, technological innovation, tractor stock, logistic model 


\section{INTRODUCCIÓN}

El tractor ha sido todo un símbolo de innovación y cambio en la agricultura. Su estudio ha tenido considerable atención de numerosos investigadores que han examinado, entre otros: la elección entre tractores y tracción animal (Lew, 2000; Olmstead y Rhode, 2001); los elementos que inciden en la evaluación económica de los tractores agrícolas (Hetz et al., 1998); el costo oportunidad, ahorro de tiempo y mejoramiento de los implementos en la adopción (Duffy y Silberberg, 2006); la proyección de la demanda (Unakitan y Akdemir, 2007). El parque de tractores agrícolas de una región o país, algunas veces es tomado como un importante indicador de la inversión en agricultura. También el grado de capitalización rural se puede observar mediante un índice estimado sobre la base de número de tractores por unidad de superficie, el que constituye un índice comparable entre regiones o países. Diversos estudios han intentado modelar el parque de tractores, donde los modelos usados han sido estimados mediante ajuste lineal (Arias, 2000; Unakitan y Akdemir, 2007) o en forma econométrica cuando se valora como maquinaria agrícola (Guadalajara-Olmeda y Fenollosa-Ribera, 2010).

La agricultura chilena adolece de estudios que apunten a generar y proponer un soporte descriptivo vinculante, que sea referente para situar los estudios de cambio técnico, social y/o económico de una región o país. Este estudio estimará la traza de la tractorización a nivel agregado, sobre la base del paradigma de crecimiento logístico, disponiendo de esta manera de una estructura referente espacio-temporal de la difusión y adopción tecnológica. En este trabajo se relaciona un balance del parque de tractores entre $t$ y $t-1$, su crecimiento en el tiempo y la evolución de la adopción agregada del tractor agrícola. De esta forma se establece un modelo que tiene la ventaja de referirse a un espacio geográfico y a un período de tiempo definido, pudiendo ser usado para fines descriptivos del proceso y también con fines de pronóstico, por ejemplo, de mercado de una región o país.

Rogers (2003) definió la difusión como el proceso mediante el cual una innovación es comunicada entre los miembros de un sistema social, mientras que adopción es el proceso que implica una decisión para hacer uso de una innovación. Karshenas y Stoneman (1995) señalaron que los modelos empíricos de adopción se han discutido bajo dos acápites: i) modelos agregados donde la variable dependiente es el número de adoptantes o la proporción de producto producido bajo una nueva tecnología, y ii) modelos desagregados donde la variable dependiente es el tiempo de adopción de la nueva tecnología por agentes individuales (Roberts y Lattin, 2000; Van Rijnsoever y Donders, 2009).

La mayoría de los modelos de adopción agregada se derivan analíticamente del comportamiento del proceso de difusión en el tiempo, cuyas características analíticas queda supeditado a la naturaleza del modelo (Rao y Kishore, 2010). Tienen su origen en el enfoque de crecimiento que ha sido adaptado para diferentes aplicaciones (Baptista, 1999; Mahajan et al., 2000; Singh, 2008, Tseng y Hu, 2009). Los resultados han mostrado un patrón temporal de adopción, cuya gráfica es una curva S o curva sigmoidea (Rao y Kishore, 2010; Calatrava y Franco, 2011).

\section{METODOLOGÍA}

\section{Tractorización}

Una de las formas de estimar el grado de mecanización de una región o país es a través del número de tractores y de la intensidad de tractorización, medida por la potencia por unidad de superficie. Numerosos estudios han probado que la tasa de crecimiento positivo de tractores, entre otros factores, han promovido el cambio técnico (Roumasset y Thapa, 1983; Hussain y Ali, 1989), siendo un indicador de prosperidad (Arias, 2000), de incremento de potencia y también de abandono de trabajadores (Unakitan y Akdemir, 2007) en el sector agrario.

\section{Balance del parque de tractores}

Para estimar el parque de tractores en un momento dado, se usa la expresión siguiente: 


$$
P_{t}^{T}=P_{t-1}^{T}+T_{t}^{N}-T_{t}^{R}
$$

Operando se tiene el balance al tiempo t:

$P_{t}^{T}-P_{t-1}^{T}=T_{t}^{N}-T_{t}^{R}$

Donde:

$P_{t}^{T}=$ Parque de tractores al momento $\mathrm{t}$.

$P_{t-1}^{T}=$ Parque de tractores al momento $\mathrm{t}-1$.

$T_{t}^{N}=$ Cantidad de tractores que se agregan al parque al momento $\mathrm{t}$.

$T_{t}^{R}=$ Cantidad de tractores que salen del parque al momento $\mathrm{t}$.

Modelo de adopción agregada

Para determinar la tasa de crecimiento del proceso de tractorización o de la difusión y adopción del tractor, se utiliza:

$$
\frac{d P^{T}(t)}{d t}=k(t)\left[S-P^{T}(t)\right]
$$

Con $P^{T}\left(t=t_{0}\right)=P_{0}^{T}$

$$
P^{T}(t)=\int_{t o}^{t} n^{T}(t) d t
$$

$n^{T}(t)$ : cantidad no acumulada de adoptantes al momento t.

$S \quad$ : cantidad máxima de adoptantes cuando se alcanza máximo crecimiento.

$k(t)$ : coeficiente de difusión.

$\frac{d P^{T}(t)}{d t}:$ Tasa en el momento t.

$P^{T}$ : cantidad acumulada de adoptantes en el momento $t_{0}$.

Relacionando con (1), se tiene:

$n_{t}^{T}=P_{t}^{T}-P_{t-1}^{T} / C_{t}^{E T}$

$C^{E T}{ }_{t:}$ cantidad de tractores por unidad adoptante al momento t.

Sea $k(t)=b P^{T}(t)$, integrando y operando algebraicamente, se obtiene (3), donde $b$ es un índice de influencia interna de la adopción (Teng et al., 2002).

$$
P^{T}=S /(1+\exp (-(-a+b t)))
$$

Los trabajos de Van den Bulte (2000), Teng et al. (2002) y Calatrava y Franco (2011) entre otros, han señalado que para caracterizar un proceso de difusión mediante el modelo de crecimiento, analíticamente expresado por la ecuación (2), es necesario conocer el origen (Po), el coeficiente de difusión $(b)$ y la condición de saturación o nivel máximo de adopción $(S)$. 


\section{Rapidez de la adopción}

Cuando se analiza un proceso de adopción mediante una curva de difusión, es importante definir un atributo que mida la rapidez de la adopción (velocidad de la sustitución tecnológica, incremento del número de adoptantes, incremento del número de ventas). Van den Bulte (2000) usó como estimador de la rapidez de la adopción, el tiempo transcurrido entre el 10 y el $90 \%$ de penetración de la innovación. Ese intervalo se puede deducir a partir de la ecuación (3), obteniéndose la ecuación (4).

$$
t_{10 \%-90 \%}=\frac{a}{b}-\frac{1}{b} \ln \left[\frac{S}{P^{T}}-1\right]=4,395 / b
$$

\section{Fuente de datos}

Se utilizaron datos provenientes de los censos agrarios entre 1930 y 1997 . Los tomos de la Dirección General de Estadística fueron la fuente para los datos de 1930 (Dirección General de Estadística, 1933) y 1936 (Dirección General de Estadística, 1938), mientras que los tomos del Servicio Nacional de Estadística y Censos de la Dirección de Estadística y Censos fueron consultados para obtener los datos para los años (SNEC, 1955) y 1965 (Dirección de Estadística y Censos, 1969), respectivamente. Los tomos del Instituto Nacional de Estadística fueron consultados para el año 1975, (INE, 1976), mientras que para el año 1997, los datos se obtuvieron de INE (1997) y del CD del Instituto Nacional de Estadísticas de Chile.

\section{Estimaciones}

La cantidad de tractores que salen del parque $\left(T^{R}\right)$ se puede estimar conociendo la vida útil, promedio del tractor. Hetz et al (1998) señalaron un periodo de vida útil que oscila entre 9 y 15 años, según el tractor proceda de un determinado grupo de países. Los tractores que se incorporan $\left(T^{N}\right)$ al parque, requiere saber de las inscripciones anuales de tractores nuevos o de las unidades que se venden anualmente en el mercado. La dificultad para obtener $T^{R}$ radica en que se obtendrán diferentes cifras finales, según sea la vida útil seleccionada, mientras que para $T^{N}$, se necesita de rigurosas estadísticas mantenidas por los registros de vehículos o de las entidades importadoras o de distribución comercial. Los datos de los censos agrarios ofrecen otra opción para superar las dificultades anteriores, puesto que representan las existencias reales de tractores $\left(P_{t}^{T}\right)$. Por tanto, una opción es estimar la curva de crecimiento con base en los datos proporcionados por los censos agrarios, como se indica más abajo.

Asimismo, se ha considerado las explotaciones agrarias informantes como las unidades adoptantes. Por definición, el adoptante es la explotación que adquiere algún tractor. Al analizar los censos de 1955, 1965, 1975 y 1997 se observó que el número medio de tractores correspondiente al stock en el conjunto de informantes era 1,76, 1,58, 1,48 y 1,47, respectivamente. Aunque solo se necesitan tres puntos para estimar una curva logística, el supuesto anterior permitió ampliar la serie disponible y permite mejorar los coeficientes de error de los parámetros de (2); se estableció el supuesto de que en los censos de 1930 y 1936, la relación tractor en uso/adoptante (informante) era de 1:1.

Se estimó la curva de crecimiento del parque y de adopción de tractores mediante el cálculo de parámetros de (3). Se aplicó un procedimiento no lineal, según el método Marquardt (Rawlings et al., 1998). Para analizar la capacidad del modelo para predecir, en el ámbito agregado (nivel país), el mercado de tractores sobre la base del número de tractores que se incorporan al parque $\left(T^{N}{ }_{t}\right)$, mediante (3) se pronosticó el parque de tractores $\left(P_{t}^{T}\right)$, para los cinco años siguientes al último de la serie (1997), luego se aplicó (1), suponiendo una vida útil de 15 años para estimar aquellos tractores que salen del parque. Para medir la aproximación del modelo a las magnitudes reales que acontecieron durante esos 5 años, se obtuvo en el RNVM (Registro Nacional de Vehículos 
Motorizados ), la cantidad de tractores inscritos y la cantidad de tractores, cuyo modelo de la marca corresponde a cada año, respectivamente. Luego, para apreciar la desviación de la predicción, se calculó el coeficiente de correlación y el error absoluto (\%), con respecto al valor estimado.

\section{RESULTADOS Y DISCUSIÓN}

Los parámetros de la curva de crecimiento (3) que describe el crecimiento del parque de tractores y del total de adoptantes se indican en el Tabla 1. Los parámetros presentaron el signo esperado y son estadísticamente significativos. Los niveles de saturación (S), pronosticado por el modelo (3), para el parque de tractores y de adoptantes (explotaciones informantes) que han adquirido el tractor, fueron de 54.799 y 37.424 , respectivamente. Los valores de S permitieron expresar los datos como proporción, para graficar la curva de crecimiento del parque de tractores y de la adopción estimada como las explotaciones informantes que adquirieron el tractor. Dado que la curva de adopción sigue la tendencia de la curva del parque de tractores, la Figura 1 presenta el patrón de la curva logística del parque de tractores y sólo indica los puntos correspondientes a las explotaciones que adquirieron el tractor en Chile entre 1930 y 1997.

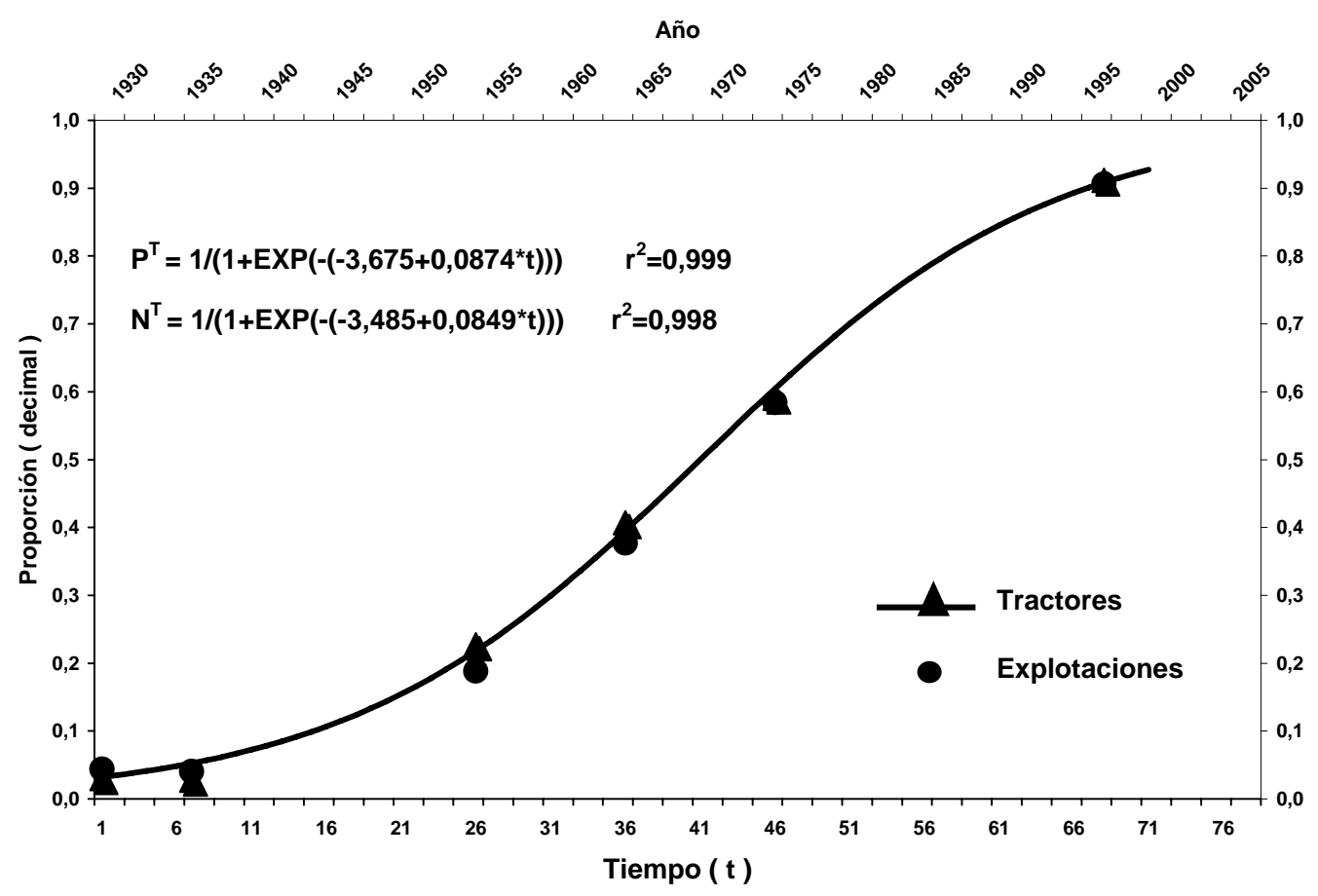

Fig. 1: Curva de crecimiento logístico del parque de tractores y puntos observados de explotaciones (informantes) como adoptantes del tractor agrícola en Chile

El propósito de un modelo de difusión, como el mostrado en la figura 1, es describir la forma en que se desarrolla en el tiempo, el grado de adopción entendido como la velocidad relativa con lo cual una innovación es adoptada por el capital humano de un sistema social (Silva y Silva, 2009), el que en este caso, está dado por los sucesivos incrementos de unidades o adoptantes. Así, el tiempo para ir de 10 a $90 \%$ de penetración del tractor en el conjunto de adoptantes del país, fue de 50 años, esto es desde 1946 a 1996 que recalculado mediante la expresión b (Tabla 1) significó un 10,1 y $89,9 \%$ de penetración. Como se esperaba, se observa que hay una relación positiva entre la cantidad de tractores que componen el parque y el número de (explotaciones informantes) adoptantes del tractor. Una extensión del modelo de difusión de influencia interna (3) estimado (Tabla 1), tiene relación con la estructura básica en términos de su punto de inflexión y la simetría, ambas propiedades matemáticas que inciden en la determinación a priori del patrón de difusión (Kauffman y Techatassanasoontornl, 2009). 
Tabla 1: Parámetros del modelo de crecimiento logístico del parque de tractores y del proceso de adopción del tractor agrícola entre 1930-1997

\begin{tabular}{|c|c|c|c|c|c|c|}
\hline Modelo & $S$ & a & $b$ & $r^{2}$ & $\begin{array}{l}\text { Error absoluto } \\
\text { medio }\end{array}$ & $\begin{array}{l}\text { Error estándar } \\
\text { estimación }\end{array}$ \\
\hline Parque Tractores & 54.799 .2 & 3.48444 & 0.0849665 & 0.998 & 597.59 & 1029.43 \\
\hline Explotaciones & 37.423 .6 & 3.67538 & 0.0874107 & 0.999 & 237.23 & 449.83 \\
\hline
\end{tabular}

Con relación a la valoración del modelo planteado para predecir el mercado de tractores en el ámbito agregado (nivel país), se correlacionó "tractores que se incorporan al parque, $\left(T^{N}{ }_{t}\right)$," estimados por (1) sobre la base de modelo a), (Tabla 1), con la cantidad de "tractores inscritos anualmente" y la cantidad de "tractores de modelo del año", registrándose coeficientes de correlación estadísticamente significativos de 0,92 y $0,90(n=5, P \leq 0.01)$, respectivamente, y el error absoluto medio (\%) de $11,9 \pm 5,8 \%$ y $17,8 \pm 2,1 \%$, respectivamente. Este simple modelo para predecir mercado a nivel agregado, también ofrece base para, indirectamente, determinar con mayor exactitud la vida útil promedio del tractor, dado que es una cifra que participa en los cálculos del balance del parque. Así, esta observación puede ofrecer un camino para futuros estudios, dada la importancia que el parámetro vida útil tiene por ejemplo cuando se valora en forma económica una maquinaria como el tractor agrícola (Hetz et al, 1998; Guadalajara-Olmeda y Fenollosa-Ribera, 2010).

\section{CONCLUSIONES}

El uso de los datos disponibles y los atributos de un modelo logístico permitieron describir la evolución del parque de tractores y la adopción, estimada sobre la base de explotaciones informantes de la existencia del tractor. La capacidad del modelo, con un significativo nivel de ajuste, se usó para predecir el nivel de saturación del parque y de la adopción y su rapidez.

El tiempo de crecimiento relevante de la adopción, dado por $\mathrm{t}_{10-90 \%}$ en el conjunto de adoptantes fue de 50 años, esto es desde 1946 (10,1\%) a $1996(89,9 \%)$.

Usando el simple modelo basado en el balance del parque de tractores vinculado a las estimaciones dadas por el modelo de crecimiento, se ensayó el pronóstico de mercado de tractores a nivel agregado, observando mérito en las magnitudes del error absoluto medido, con relación a la serie de contraste (Tractores totales anuales y modelo del año, inscritos en RNVM). También ofrece una metodología que, en forma indirecta, busque estimar la vida útil del tractor en una región o país.

El modelo describió el crecimiento de la adopción de una innovación tecnológica, dado por el tractor agrícola, pero carece de explicación acerca de los factores que explican su crecimiento, aspecto que puede ofrecer un camino de estudio.

\section{AGRADECIMIENTOS}

Los autores agradecen a la Vicerrectoría de Investigación y Desarrollo (VRID) de la Universidad de Santiago de Chile (USACH) por el financiamiento otorgado para la ejecución del Proyecto DICYT-030674MC del que se ha derivado este trabajo.

\section{REFERENCIAS}

Arias M., P., El proceso de mecanización en la actividad agrícola. Estudios Agrosociales y Pesqueros: 187, 9-38 (2000). 
Baptista, R., The diffusion of process innovations: A selective review. International Journal of the Economics of Business: 6(1), 107-129 (1999).

Calatrava, J. y J.A. Franco, Using pruning residues as mulch: Analysis of its adoption and process of diffusion in Southern Spain olive orchards. Journal of Environmental Management 92, 620-629 (2011).

Dirección de Estadística y Censos; IV Censo Nacional Agropecuario, año agrícola 19641965. Dirección de Estadística y Censos, República de Chile (1969).

Dirección General de Estadística; Censo Agropecuario 1929-1930. Dirección General de Estadística, República de Chile (1933).

Dirección General de Estadística; Agricultura 1935/36 Censo. Dirección General de Estadística de Chile. República de Chile (1938).

Duffy M., D. y E. Silberberg, The diffusion of tractor technology. The Journal of Economic History: 66(2), 354-389 (2006).

Guadalajara-Olmeda, N. y M.L. Fenollosa-Ribera, Modelos de valoración de maquinaria agrícola en el Sur de Europa. Un análisis de depreciación real. Agrociencia: 44; 381-391 (2010).

Hetz, E., A. Huerta, S. Villar y M. López, Evaluación económica de los tractores agrícolas comercializados en Chile. Agro sur: 26(2), 33-43 (1998).

Hussain, A., y F. Ali, Impact of tractorization on employment and productivity in NWFP. Peshwar (Pakistan): Institute of Development Studies, N.W.F.P. Agricultural University (1989).

INE, V Censo Nacional Agropecuario 1975-1976. Instituto Nacional de Estadísticas, Ministerio de Economía, Fomento y Reconstrucción, República de Chile (1976).

INE, Estadísticas agropecuarias 1997. Disponible en http://www.ine.cl acceso el 20 de julio de 2008.

Karshenas, M. y P. Stoneman, Technological diffusion. In: Handbook of the economics of innovation and technological change. p. 265-297. Stoneman, P. (ed.). Blackwell Handbooks in Economics, Blackwell Publishing. Oxford. GB. 608 p. (1995).

Kauffman, R.J., A. y A. A. Techatassanasoontorn, Understanding early diffusion of digital wireless phones, Telecommunications Policy: 33, 432-450 (2009)

Lew, B., The diffusion of tractors on the Canadian prairies: The Threshold model and the problem of uncertainty. Explorations in Economic History: 37(2),189-216 (2000).

Mahajan, V., E. Muller, y Y. Wind, New product diffusion models: From theory to practice. In: Mahajan, V., Muller, E., Wind, Y. (Eds.) New product diffusion models. International series in quantitative marketing:11. Kluwer Academic Publishers. p. 3-24 (2000).

Olmstead, A.L. y P.W. Rhode, Reshaping the landscape: The impact and diffusion of the tractor in american agriculture, 1910-1960, The Journal of Economic History: 61(3), 663-698 (2001).

Rao, K.U. y V.V.N. Kishore, A review of technology diffusion models with special reference to renewable energy technologies, Renewable and Sustainable Energy Reviews: 14, 1070-1078 (2010).

Rawlings, J.O., S.G. Pantula and D.A Dickey (eds), Applied regression analysis. A research tool. Second Edition, Springer -Verlag, New York, NY. 486 p. (1998). 
Roberts, J.H. y J.M. Lattin, Disaggregate-level diffusion models. In: Mahajan, V., Muller, E. and Wind, Y. (eds.) New-products diffusion models. International series in quantitative marketing, Kluwer Academic Publishers p. 207-236 (2000).

Rogers, E.M., Diffusions of innovations, Fifth Edition, The Free Press, Collier Macmillan Publishers, London. 453 p. (2003).

Roumasset, J. y G. Thapa, Explaining tractorization in Nepal: An alternative to the consequences approach. Journal of Development Economics: 12(3), 377-395 (1983).

SNEC, III Censo Nacional Agrícola Ganadero. Servicio Nacional de Estadística y Censos, República de Chile (1955).

Silva, P. y R. Silva, Asimilación del Almacén de Datos en las Organizaciones Corporativas Petroquímicas. Información Tecnológica, ISSN: 0718-0764 (en línea), 20(2):41-46, 2009. http://www.scielo.cl/scielo.php?script=sci_arttext\&pid=S0718-

07642009000200006\&lng=es\&nrm=iso>. doi: 10.4067/S0718-07642009000200006).Acceso: 2 de Mayo (2011)

Singh, S.K., The diffusion of mobile phones in India. Telecommunications Policy: 32, 642-651 (2008).

Tseng, F.M. y Y.C. Hu. Quadratic-interval Bass model for new product sales diffusion. Expert Systems with Applications: 36, 8496-8502 (2009)

Teng, J.T.C, V. Grover, y W. Güttler, Information technology innovations: General diffusion patterns and its relationships to innovation characteristics. IEEE Transactions on Engineering Management: 49(1), 13-27 (2002).

Unakitan, G. y B. Akdemir. Tractor demand projection in Turkey. Biosystems Engineering: 97, 1925 (2007).

Van den Bulte, C., New product diffusion acceleration: measurement and analysis. Marketing Science 19(4):366-380 (2000).

Van Rijnsoever, F.J. y A. R. Donders. The effect of innovativeness on different levels of technology adoption. Journal of the American Society for Information Science and Tecnology: 60(5):984-996 (2009). 